\title{
POSICIONAMENTO CIRÚRGICO: PREVALÊNCIA DE RISCO DE LESÕES EM PACIENTES CIRÚRGICOS
}

\author{
Surgical positioning: prevalence of risk of injuries in surgical patients
}

\author{
Posicionamento quirúrgico: prevalencia de riesgo de lesiones en pacientes quirúrgicos
}

Denilse Damasceno Trevilato ${ }^{1 *}$, Thaís Camargo de Meloํ․ Maria Anobes Bonet Grespan Fagundes ${ }^{3}$, Rita Catalina Aquino Caregnato ${ }^{4}$

RESUMO: Objetivo: Determinar a prevalência de pacientes em risco de desenvolvimento de lesões decorrentes do posicionamento cirúrgico. Método: Estudo transversal, realizado em hospital privado localizado na região sul do Brasil. Amostra aleatória com 378 pacientes adultos submetidos a cirurgias eletivas entre janeiro e setembro de 2017. Foi aplicada a Escala de Avaliação de Risco para o Desenvolvimento de Lesões Decorrentes do Posicionamento Cirúrgico (ELPO) após indução anestésica e realizada análise descritiva. Resultados: A prevalência de pacientes com alto risco de desenvolvimento de lesões foi de $19,05 \%$ ( $n=72$ ). O posicionamento identificado como de maior risco foi a litotomia $(59,72 \%$; $=43)$. O escore médio da ELPO na amostra estudada foi 16,317 (desvio padrão=3,6176) e a mediana foi de 16, o que significa baixo risco de desenvolvimento de lesões. Conclusão: A ELPO permitiu determinar a prevalência de risco para lesões em pacientes submetidos a procedimentos eletivos, identificando que o risco está mais relacionado com a posição cirúrgica do que com o porte da cirurgia.

Palavras-chave: Posicionamento do paciente. Medição de risco. Período intraoperatório. Enfermagem perioperatória. Ferimentos e lesões.

ABSTRACT: Objective: To determine the prevalence of patients at risk of developing lesions due to surgical positioning. Method: A cross-sectional study was carried out in a private hospital in southern Brazil. Randomized sample with 378 adult patients submitted to elective surgeries between January and September 2017. The Risk Assessment Scale for the Development of Injuries due to Surgical Positioning (ELPO) was used after anesthetic induction and a descriptive analysis was performed. Results: The prevalence of patients at high risk of developing lesions was $19.05 \%$ ( $\mathrm{n}=72$ ). The lithotomic position was identified as the one with greatest risk (59.72\%; $\mathrm{n}=43)$. The mean ELPO score in the sample was 16.317 (standard deviation=3.6176) and the median was 16, meaning low risk of developing lesions. Conclusion: ELPO allowed to determine the prevalence of risk for lesions in patients submitted to elective procedures, identifying that the risk is more related to surgical position than to the size of the surgery. Keywords: Patient positioning. Risk assessment. Intraoperative period. Perioperative nursing. Wounds and injuries.

RESUMEN: Objetivo: Determinar la prevalencia de pacientes en riesgo de desarrollo de lesiones derivadas del posicionamento quirúrgico. Método: Estudio transversal, realizado em um hospital privado em el Sur de Brasil. Muestra aleatoria con 387 pacientes adultos sometidos a cirugías electivas entre enero y septiembre de 2017. Se aplicó la Escala de Evaluación de Riesgo para el Desarrollo de Lesiones Transcurrentes del Posicionamiento Quirúrgico (ELPO) después de la inducción anestésica y análisis descriptivo. Resultados: La prevalencia de pacientes con alto riesgo de desarrollo de lesiones fue del 19,05\% $(\mathrm{n}=72)$. El posicionamiento identificado como de mayor riesgo fue la litotomía $(59,72 \%, \mathrm{n}=43)$. El score promedio de la ELPO en la muestra estudiada fue 16,317 (desviación estándar=3,6176) y la mediana fue de 16, lo que significa bajo riesgo de desarrollo de lesiones. Conclusión: La ELPO permitió determinar la prevalencia de riesgo para lesiones en pacientes sometidos a procedimientos electivos, identificando que el riesgo está más relacionado con la posición quirúrgica que con el porte de la cirugía.

Palabras clave: Posicionamiento del paciente. Medición de riesgo. Periodo intraoperatorio. Enfermería perioperatoria. Heridas y lesiones.

'Enfermeira; mestranda em Ensino na Saúde da Universidade Federal de Ciências da Saúde de Porto Alegre (UFCSPA). Enfermeira do Centro Cirúrgico do Hospital Moinhos de Vento - Porto Alegre (RS), Brasil. Enfermeira do Centro Cirúrgico do Hospital Moinhos de Vento - Porto Alegre (RS), Brasil.

${ }^{3}$ Enfermeira; mestre em Ensino na Saúde. Enfermeira do Centro de Recuperação Pós-Anestésica do Hospital Moinhos de Vento - Porto Alegre (RS), Brasil.

¿Enfermeira; mestra em Enfermagem; doutora em Educação. Professora do curso de graduação em Enfermagem da UFCSPA - Porto Alegre (RS), Brasil.

*Autora correspondente: denilset@hotmail.com

Recebido: 28/11/2017 - Aprovado: 15/04/2018

DOI: $10.5327 / Z 1414-4425201800030003$ 


\section{INTRODUÇÃo}

O posicionamento cirúrgico ideal para o paciente deve ser o mais anatômico e fisiológico possível, mantendo o alinhamento corporal, com o mínimo de tensão e pressão sobre o tecido, preservando as funções ventilatória e circulatória e evitando exposição desnecessária, além de permitir ao cirurgião um bom acesso ao sítio cirúrgico e, ao anestesista, acesso às linhas de infusão e monitorização ${ }^{1}$. Toda a equipe (anestesista, cirurgião e enfermagem) deve se envolver com esse processo logo após a indução anestésica para proporcionar um posicionamento seguro e confortável do paciente.

Lesões por pressão (LP) decorrentes do posicionamento cirúrgico são consideradas eventos adversos, causados devido ao procedimento cirúrgico. O ideal é que se identifique e previna danos evitáveis, por meio da criação de métricas e padrões. Por esse motivo, a mensuração desse evento é fundamental para a melhoria da assistência ao paciente cirúrgico ${ }^{2}$.

Um estudo validou uma escala de avaliação de risco de desenvolvimento de leões provocadas por posicionamento cirúrgico, que foi aplicada a uma amostra de 115 pacientes submetidos a procedimentos cirúrgicos, constatando desenvolvimento de LP em 25 deles (21,7\%), sendo que $46(40,0 \%)$ apresentaram dor relacionada ao posicionamento cirúrgico no pós-operatório ${ }^{3}$. Dos pacientes com lesão, 3 tinham LP previamente ao procedimento, ocasionando uma evolução; em 2 foram identificadas LP no período imediatamente após o procedimento cirúrgico e 20 pacientes tiveram a identificação da LP durante as 72 horas subsequentes ${ }^{3}$.

Observa-se uma discrepância nos resultados encontrados em estudos sobre as lesões por posicionamento cirúrgico. Outro estudo retrospectivo, que avaliou 38 mil procedimentos em prontuários, encontrou registros de 40 lesões, com prevalência de $0,1 \%{ }^{4}$. Um estudo longitudinal com 199 pacientes evidenciou uma incidência de $20,6 \%$ de lesões ${ }^{5}$. Essa questão é preocupante, visto que as lesões podem ser transitórias ou permanentes, aumentando o tempo de internação e prolongando a recuperação do paciente ${ }^{6}$.

Dependendo da posição cirúrgica, da anestesia e do tempo de duração da cirurgia, o paciente poderá ter o risco aumentado de lesões por posicionamento e somam-se a isso a idade e as comorbidades. Os enfermeiros do centro cirúrgico (CC) devem estar atentos para identificar os pacientes de risco e evitar eventos adversos decorrentes do posicionamento ${ }^{7,8}$.

As posições básicas que originam o posicionamento cirúrgico são três: supina ou decúbito dorsal; prona ou decúbito ventral; e lateral. Cada posição pode derivar em outras, incluindo algumas alterações, tais como: elevação dos joelhos, adução ou abdução dos membros inferiores ou superiores e céfalo-aclive ou céfalo-declive (Trendelenburg), entre outras 9 . A prona é a mais desafiadora para a equipe cirúrgica porque o paciente é usualmente colocado nessa posição após ser anestesiado, não tendo como sinalizar qualquer desconforto decorrente da posição ou se reacomodar durante a cirurgia ${ }^{1,10}$.

A decisão final sobre o posicionamento do paciente geralmente é do cirurgião, entretanto, o enfermeiro assistencial deve participar desse processo e agir como advogado do paciente sempre que algum fator estiver interferindo na sua segurança ${ }^{1}$.

Estratégias devem ser adotadas para a diminuição do risco de lesão por posicionamento, como o uso de superfícies de suporte, espuma, gel e o reposicionamento transoperatório, sempre que o procedimento permitir ${ }^{8,11}$.

A escala de Braden é preditiva de risco de LP, mas é utilizada para pacientes clínicos, não sendo indicada durante o procedimento cirúrgico, visto que não avalia fatores específicos, como tempo cirúrgico, anestesia e comorbidades ${ }^{8}$.

Em 2013, um instrumento foi criado e validado para avaliar o risco de desenvolvimento de lesões e fornecer subsídios para a melhoria na assistência da enfermagem ao intraoperatório por meio do desenvolvimento de protocolos voltados ao posicionamento do paciente ${ }^{3}$. A Escala de Avaliação de Risco para Desenvolvimento de Lesão Decorrente do Posicionamento Cirúrgico do Paciente (ELPO) demonstrou ser um instrumento válido para avaliação de riscos aos pacientes, para prever tanto desfechos como LP como a presença de dor no pós-operatório decorrente do posicionamento cirúrgico. Por ser um instrumento novo de avaliação de risco de lesão por posicionamento, torna-se necessário ampliar o escopo da sua utilização, realizando outras pesquisas em diferentes contextos hospitalares ${ }^{3}$.

Desta forma, as autoras deste artigo, enfermeiras com atuação em CC, consideraram importante conhecer o perfil de pacientes atendidos em uma instituição privada, traçando o problema de pesquisa, e investigar qual é a prevalência de pacientes em risco de desenvolvimento de lesões decorrentes do posicionamento cirúrgico.

\section{OBJETIVO}

Determinar a prevalência de pacientes com risco de desenvolvimento de lesões decorrentes do posicionamento cirúrgico. 


\section{MÉTODO}

Estudo transversal, realizado em um hospital privado geral, de grande porte, localizado em Porto Alegre, Rio Grande do Sul, Brasil. O CC dispõe de 17 salas operatórias, nas quais foram realizadas 22.129 cirurgias no ano de 2016 .

A amostra foi probabilística aleatória, constituída por 378 pacientes submetidos a procedimentos cirúrgicos. Para o cálculo amostral, considerou-se o risco maior de desenvolvimento de lesões transoperatórias, utilizando-se o programa WINPEPI for Windows, versão 11.43, desenvolvido por Paul M. Gahlinger, com margem de confiança de $95 \%$, margem de erro de $5 \%$ e proporção de $50 \%$. Os critérios de inclusão foram: idade igual ou superior a 18 anos e com cirurgia eletiva agendada, independentemente da especialidade cirúrgica.

Uma das pesquisadoras capacitou sete enfermeiras do CC para aplicar a escala ELPO nos pacientes atendidos nos três turnos (manhã, tarde e noite). O período de coleta de dados foi de janeiro a setembro de 2017.

A coleta de dados ocorreu diariamente, com o sorteio dos pacientes a partir da escala cirúrgica informatizada. Utilizando o programa Microsoft ${ }^{\circledR}$ Excel, criou-se na escala uma coluna com números aleatórios para cada cirurgia, que foram ordenados de forma crescente, sendo selecionados os primeiros sete pacientes da lista. Na sala de admissão do CC, os pacientes previamente sorteados eram abordados pelo enfermeiro ou técnico de enfermagem, que explicava o objetivo da pesquisa e investigava o interesse em participar do estudo. No caso de aceite, era fornecido o Termo de Consentimento Livre e Esclarecido (TCLE), em duas vias, ficando uma com o paciente. Em seguida, o paciente era conduzido à sala operatória $(\mathrm{SO})$, posicionado e realizava-se a indução anestésica. Somente após essas etapas concluídas a enfermeira avaliava o paciente e preenchia uma planilha manual com dados referentes à escala ELPO; posteriormente, os dados eram digitados em uma planilha Excel.

A escala ELPO sugere um ponto de corte, pelo qual pacientes com escore igual ou menor a 19 são considerados com risco menor para o desenvolvimento de lesões decorrentes do posicionamento cirúrgico; e pacientes com escore igual ou maior que 20 são considerados com risco maior ${ }^{3}$. A análise realizou-se por meio de estatística descritiva, apresentada em proporção, mediana, média e desvio padrão (DP).

A pesquisa foi registrada na Plataforma Brasil e aprovada pelo Comitê de Ética e Pesquisa da Instituição, com o CAAE número 59023916.6.0000.5330.

\section{RESULTADOS}

Em relação ao perfil cirúrgico da amostra, identificou-se: 259 pacientes do sexo feminino (68,52\%); 199 como ASA (American Society of Anesthesiology) II em relação ao risco anestésico $(52,64 \%)$ e 159 foram submetidos a procedimentos de porte médio $(42,06 \%)$ (Tabela 1$)$.

O escore médio da aplicação da escala ELPO na amostra investigada $(\mathrm{n}=378)$ foi de $16,317(\mathrm{DP}=3,6176)$, mediana de 16, com escore mínimo de 7 e máximo de 26.

Observa-se, na Tabela 2, que 209 pacientes $(55,29 \%)$ permaneceram em posição supina ou dorsal durante o procedimento cirúrgico; e para 276 deles $(73,01 \%)$ a cirurgia teve duração de até 2 horas. Em 170 pacientes (44,97\%) utilizaramse coxins de campos de algodão. A posição anatômica foi adotada em $70(18,51 \%)$ e a abertura dos membros superiores, no máximo $90^{\circ}$, em $175(46,30 \%)$. Verificou-se que 234 pacientes $(61,69 \%)$ não tinham comorbidades que pudessem aumentar o risco de lesões decorrentes do posicionamento.

Pela aplicação da escala ELPO, identificou-se 72 cirurgias com maior risco para lesões decorrentes do posicionamento, sendo essas classificadas quanto ao porte cirúrgico:

Tabela 1. Distribuição dos pacientes cirúrgicos $(n=378)$ de acordo com sexo, classificação ASA, porte cirúrgico e Escala de Avaliação de Risco para Desenvolvimento de Lesão Decorrente do Posicionamento Cirúrgico do Paciente, atendidos no centro cirúrgico de um hospital privado. Porto Alegre (RS), 2017.

\begin{tabular}{|l|c|}
\hline Variáveis & $\begin{array}{c}\text { Frequência } \\
\mathbf{n}(\%)\end{array}$ \\
\hline Sexo & $119(31,48)$ \\
\hline Masculino & $259(68,52)$ \\
\hline Feminino & \\
\hline ASA & $162(42,86)$ \\
\hline I & $199(52,64)$ \\
\hline II & $17(4,50)$ \\
\hline III & $151(39,95)$ \\
\hline Porte cirúrgico & $159(42,06)$ \\
\hline Pequeno & $68(17,99)$ \\
\hline Médio & \\
\hline Grande & $306(80,95)$ \\
\hline Classificação por ELPO & $72(19,05)$ \\
\hline Baixo risco
\end{tabular}

ELPO: Escala de Avaliação de Risco para Desenvolvimento de Lesão Decorrente do Posicionamento Cirúrgico do Paciente. 
Tabela 2. Distribuição das variáveis avaliadas por meio da Escala de Avaliação de Risco para Desenvolvimento de Lesão Decorrente do Posicionamento Cirúrgico do Paciente em pacientes atendidos no centro cirúrgico de um hospital privado. Porto Alegre (RS), 2017.

\begin{tabular}{|c|c|}
\hline Variáveis da ELPO & $\begin{array}{l}\text { Frequência } \\
\text { n (\%) }\end{array}$ \\
\hline \multicolumn{2}{|l|}{ Idade do paciente (anos) } \\
\hline Entre 18 e 39 & $115(30,42)$ \\
\hline Entre 40 e 59 & $150(39,68)$ \\
\hline Entre 60 e 69 & $75(19,84)$ \\
\hline Entre 70 e 79 & $24(6,35)$ \\
\hline Acima de 80 & $14(3,71)$ \\
\hline \multicolumn{2}{|l|}{ Comorbidades } \\
\hline Sem comorbidades & $234(61,90)$ \\
\hline Doença vascular & $88(23,28)$ \\
\hline Diabetes mellitus & $08(2,12)$ \\
\hline Obesidade ou desnutrição & $47(12,44)$ \\
\hline UP ou neuropatia previamente diagnosticada & $01(0,26)$ \\
\hline \multicolumn{2}{|l|}{ Tempo de duração da cirurgia (horas) } \\
\hline Até 1 hora & $139(36,77)$ \\
\hline De 1 até 2 & $137(36,24)$ \\
\hline De 2 até 4 & $89(23,55)$ \\
\hline De 4 até 6 & $12(3,18)$ \\
\hline Acima de 6 & $01(0,26)$ \\
\hline \multicolumn{2}{|l|}{ Tipo de anestesia } \\
\hline Local & $39(10,32)$ \\
\hline Sedação & $71(18,78)$ \\
\hline Regional & $42(11,11)$ \\
\hline Geral & $217(57,41)$ \\
\hline Geral + regional & $09(2,38)$ \\
\hline \multicolumn{2}{|l|}{ Superfície de suporte } \\
\hline $\begin{array}{l}\text { Colchão de viscoelástico + coxins de } \\
\text { viscoelástico }\end{array}$ & $120(31,75)$ \\
\hline Colchão de espuma + coxins de viscoelástico & - \\
\hline Colchão de espuma + coxins de espuma & $88(23,28)$ \\
\hline $\begin{array}{l}\text { Colchão de espuma + coxins de campos de } \\
\text { algodão }\end{array}$ & $170(44,97)$ \\
\hline $\begin{array}{l}\text { Sem uso de superfícies de suporte ou } \\
\text { suportes rígidos sem acolchoamento ou } \\
\text { perneiras estreitas }\end{array}$ & - \\
\hline \multicolumn{2}{|l|}{ Posição dos membros } \\
\hline Posição anatômica & $70(18,51)$ \\
\hline Abertura $<90^{\circ}$ dos membros superiores & $175(46,30)$ \\
\hline $\begin{array}{l}\text { Elevação dos joelhos }<90^{\circ} \text { e abertura dos } \\
\text { membros inferiores }<90^{\circ} \text { ou pescoço sem } \\
\text { alinhamento esternal }\end{array}$ & $115(30,42)$ \\
\hline $\begin{array}{l}\text { Elevação dos joelhos }>90^{\circ} \text { ou abertura dos } \\
\text { membros inferiores }>90^{\circ}\end{array}$ & $12(3,17)$ \\
\hline $\begin{array}{l}\text { Elevação dos joelhos }>90^{\circ} \text { e abertura dos } \\
\text { membros inferiores }>90^{\circ} \text { ou abertura dos } \\
\text { membros superiores }>90^{\circ}\end{array}$ & $06(1,60)$ \\
\hline
\end{tabular}

ELPO: Escala de Avaliação de Risco para Desenvolvimento de Lesão Decorrente do Posicionamento Cirúrgico do Paciente; UP: Úlcera por Pressão.
22 de pequeno porte, 22 de médio porte e 28 de grande porte. Dessas, em relação ao posicionamento: 43 pacientes $(59,72 \%)$ estavam em posição litotômica, 14 (19,44\%) em decúbito dorsal, 9 (12,50\%) em prona, 4 (5,56\%) em decúbito lateral e $2(2,78 \%)$ em Trendelenburg.

\section{DISCUSSÃO}

Na amostra investigada, obteve-se um escore médio na escala ELPO de 16, o que significa baixo risco para desenvolvimento de lesões decorrentes do posicionamento cirúrgico ${ }^{3}$. No CC pesquisado, mensalmente, são realizadas em média 2 mil cirurgias, sendo aproximadamente $80 \%$ de pequeno e médio porte. Das 72 cirurgias identificadas com maior risco para lesões decorrentes do posicionamento, 22 eram de pequeno porte, 22 de médio porte e 28 de grande porte, portanto, verificou-se que o risco de lesão não estava diretamente relacionado ao porte.

O posicionamento é um dos fatores fundamentais para a realização de um procedimento seguro e efetivo. Ao posicionar-se o paciente, é necessário ter cuidado com as articulações dos quadris, joelhos e membros superiores e inferiores, pois lesões nervosas podem ocorrer ${ }^{6}$ caso a abertura ou flexão das extremidades sejam maiores que $90^{\circ}$. Em relação à posição dos membros, nos critérios de avaliação apresentados na ELPO, identificou-se que 95,23\% da amostra foi posicionada dentro dos limites aceitos para abertura e flexão.

Observa-se, na Tabela 2, que as posições cirúrgicas mais utilizadas foram supina ou dorsal $(55,29 \%)$ e litotômica $(22,75 \%)$. A posição supina é mais anatômica. Nela, o aumento da pressão visceral abdominal sobre a veia cava inferior reduz o retorno do sangue venoso ao coração ${ }^{7,12}$. As complicações relacionadas a essa posição ocorrem devido ao posicionamento inadequado e ao tempo prolongado ${ }^{13}$. Na posição litotômica, o paciente é posicionado em decúbito dorsal, com os membros inferiores abduzidos, apoiados sobre um suporte de pernas e elevados, formando um ângulo de aproximadamente $90^{\circ} \mathrm{com}$ a articulação do quadril. Essa posição oferece maior risco de complicações devido à pressão nas regiões sacra e lombar ${ }^{9}$, portanto, devese utilizar uma proteção específica, como cobertura adesiva ou coxin de viscoelástico ${ }^{14}$. A pressão do suporte nas pernas e pés pode trazer danos ao nervo fibular, causando a "queda" plantar. Quanto maior a flexão dos membros inferiores sobre o quadril, maior será a pressão intra-abdominal, diminuindo a expansão pulmonar ${ }^{12}$. Essa posição 
pode causar complicações para qualquer paciente, porém, nos idosos, desnutridos e obesos ${ }^{6,9}$ são mais graves e frequentes ${ }^{13}$. Em relação ao posicionamento nas 72 cirurgias identificadas com maior risco para desenvolvimento de lesão, evidenciou-se que: 43 pacientes $(59,72 \%)$ estavam em posição litotômica; 14 (19,44\%) em decúbito dorsal; 9 (12,5\%) em prona; 4 (5,56\%) em decúbito lateral e 2 (2,78\%) em Trendelenburg. Observou-se, neste estudo, que os pacientes em posição litotômica foram, em sua maioria, classificados com maior risco para lesão.

Um estudo realizado com o objetivo de avaliar a incidência de lesão decorrente do posicionamento cirúrgico e apontar os fatores de risco identificou lesões em 12,20\%, dos 172 pacientes avaliados. Dos pacientes com lesões, 90,50\% foram classificados como ASA II e ASA III ${ }^{15}$. Os dados contidos na Tabela 1 mostram $57,14 \%$ da amostra com classificação de risco anestésico similar ao estudo citado. Pacientes ASA III ou mais têm maior risco para o desenvolvimento de lesões ${ }^{8}$. No presente estudo, apenas $4,5 \%$ foram identificados como ASA III.

Lesões por posicionamento ocorrem com uma frequência três vezes maior em pacientes submetidos a cirurgias com mais de duas horas ${ }^{15}$. Com relação ao tempo cirúrgico, 102 procedimentos $(26,99 \%)$ tiveram duração maior que 2 horas e, para 276 pacientes $(73,01 \%)$ os procedimentos duraram 2 horas ou menos, com menor risco de lesões.

Dois estudos sobre lesões e fatores de risco associaram a anestesia geral à maior ocorrência de lesões, pelo fato de reduzir a sensibilidade. Essa é a técnica de escolha em cirurgias de grande porte com maior duração e verificou-se incidência de lesão de $85,70^{15}$ e $75 \%{ }^{4}$ nos pacientes submetidos a esse método anestésico. No presente estudo, a anestesia geral foi utilizada em 217 pacientes $(57,41 \%)$.

Em relação às superfícies de suporte utilizadas no posicionamento de pacientes cirúrgicos, os lençóis e cobertores diminuem a sua eficácia ${ }^{8}$. Na amostra estudada, foram utilizados coxins de campo de algodão em 170 pacientes (44,97\%) e em 120 (31,75\%), colchão ou coxins de viscoelástico. Esses dispositivos oferecem mais benefícios aos pacientes, principalmente aos idosos e em cirurgias com mais de duas horas de duração ${ }^{9}$. Na instituição na qual este estudo foi realizado, os colchões de todas as mesas cirúrgicas são de viscoelástico.

Um estudo sobre fatores de risco para desenvolvimento de LP evidenciou maior incidência na faixa entre 38 e 58 anos $(40,60 \%)^{16}$. No entanto, em outra pesquisa esse mesmo desfecho ocorreu em pacientes na faixa etária entre 45 e 64 anos
$(52,40 \%)^{15}$. Pesquisas demonstram que a idade tem influência sobre risco de desenvolvimento de lesões, entretanto, não deve ser um critério isolado de avaliação ${ }^{16-18}$. Com relação à idade dos pacientes da amostra avaliada, a maioria estava na faixa entre 40 e 59 anos (39,68\%).

Doenças vasculares predispõem a ocorrência de LP, sendo que $34 \%$ dos pacientes que desenvolveram lesão tinham hipertensão arterial sistêmica ${ }^{4}$ e esse risco aumenta quando associado a outras comorbidades e idade avançada $^{15}$. Em pacientes com índice de massa corporal menor que 20 ou maior de 30 , o sobrepeso e a magreza aumentam a fricção e o cisalhamento ${ }^{6,16,19}$. Na amostra estudada, 88 pacientes $(23,28 \%)$ eram portadores de doença vascular e $47(12,44 \%)$ tinham obesidade ou desnutrição. Entretanto, $234(61,90 \%)$ não apresentavam nenhuma comorbidade associada ao aumento de risco de lesão, portanto, a maioria possuía menor risco.

Em relação às limitações do estudo, destaca-se que foi realizado em uma única instituição, não tendo sido mensurado o desfecho lesão decorrente do posicionamento. Sugere-se, para estudos futuros, que sejam aplicados em outros cenários hospitalares, bem como que se faça o acompanhamento e o registro da ocorrência de lesões nos pacientes avaliados com alto risco pela escala ELPO.

\section{CONCLUSÃO}

A aplicação da escala ELPO permitiu determinar uma prevalência de $19,05 \%$ de pacientes submetidos a procedimentos eletivos com risco maior de desenvolvimento de lesões decorrentes do posicionamento cirúrgico, identificando-se que o risco está mais relacionado com a posição cirúrgica do que com o porte da cirurgia. O escore médio da ELPO foi de 16, indicando que a amostra estudada foi composta por pacientes com menor risco.

Considera-se importante este diagnóstico, pois permite ao enfermeiro direcionar o planejamento do cuidado. A escala mostrou-se eficaz para qualificar o atendimento do paciente no intraoperatório.

Sugere-se a utilização da escala de ELPO como protocolo de avaliação de risco de lesão para todos os pacientes cirúrgicos, bem como a substituição dos coxins de espuma e algodão por coxins de viscoelástico. Pesquisas futuras poderão mensurar o desfecho de dor musculoesquelética e lesões decorrentes do posicionamento cirúrgico no pós-operatório imediato, relacionando esses eventos à avaliação de risco. 


\section{REFERÊNCIAS}

1. Spruce L, Van Wicklin AS. Back to basics: positioning the patient. AORN J [Internet]. 2014 set. [citado em 10 out. 2017];100(3):298-305. Disponível em: https://www.aorn.org/websitedata/cearticle/pdf_file/ CEA14530-0001.pdf http://dx.doi.org/10.1016/j.aorn.2014.06.004

2. National Patient Safety Foundation. Free from harm: accelerating patient safety improvement fifteen years after To Err Is Human [Internet]. Boston: National Patient Safety Foundation; 2015 [citado em 02 nov. 2017]. Disponível em: https://www.aig.com/content/dam/aig/americacanada/us/documents/brochure/free-from-harm-final-report.pdf

3. Lopes CMM, Haas VL, Dantas RAS, Oliveira CG, Galvão CM. Assessment scale of risk for surgical positioning injuries. Rev Latinoam Enferm [Internet]. 2016 ago. [citado em 30 jul. 2017];24:e2704. Disponível em: http://www.scielo.br/scielo. php?script=sci_arttext\&pid=S0104-11692016000100395 http://dx.doi.org/10.1590/1518-8345.0644.2704

4. Saraiva IL, Paula MDFC, Carvalho R. Úlcera por pressão no período transoperatório: ocorrência e fatores associados. Rev SOBECC [Internet]. 2014 [citado em 12 jul. 2017];19(4):207-13. Disponivel em: http://sobecc. org.br/arquivos/artigos/2015/pdfs/v19n4/SOBECC_v19n4_207-213.pdf http://dx.doi.org/10.5327/Z1414-4425201400040006 ARTIGO ORIGINAL

5. Scarlatti KC, Michel JLM, Gamba MA, Gutiérrez MGR. Úlcera por pressão em pacientes submetidos à cirurgia: incidência e fatores associados. Rev Esc Enferm USP [Internet]. 2011 [citado em 22 ago. 2017];45(6):1372-9. Disponivel em: http://www.scielo.br/pdf/reeusp/v45n6/v45n6a14.pdf

6. Fleisch MC, Bremerich D, Schulte-Mattler W, Tannen A, Teichmann AT, Bader W, et al. The prevention of positioning injuries during gynecologic operations guideline of DGGG (S1-Level, AWMF Registry No. 015/077, February 2015). Geburtshilfe Frauenheilkd [Internet]. 2015 ago. [citado em 10 out. 2017];75(8):792-807. Disponível em: https:/www.ncbi.nlm.nih.gov/pmc/articles/PMC4554497/ https://dx.doi.org/10.1055\%2Fs-0035-1557776

7. Lopes CMDM, Galvão CM. Surgical positioning: evidence for nursing care. Rev Latinoam Enferm [Internet]. 2010 [citado em 03 jun. 2017];18(2):287-94. Disponível em: http://www.scielo.br/ scielo.php?script=sci_arttext\&pid=S0104-11692010000200021 http://dx.doi.org/10.1590/S0104-11692010000200021

8. Spruce L. Back to basics: preventing perioperative pressure injuries. AORN J [Internet]. 2017 [citado em 12 out. 2017];105(1):92-9. Disponível em: https:// aornjournal.onlinelibrary.wiley.com/doi/full/10.1016/j.aorn.2016.10.018 https://doi.org/10.1016/j.aorn.2016.10.018

9. Associação Brasileira de Enfermeiros de Centro Cirúrgico, Recuperação Anestésica e Centro de Material e Esterilização (SOBECC). Diretrizes de práticas em enfermagem cirúrgica e processamento de produtos para a saúde. 7ª ed. São Paulo: SOBECC; 2017.
10. Salkind EM. A novel approach to improving the safety of patients undergoing lumbar laminectomy. AANA J [Internet]. 2013 [citado em 10 out. 2017];81 (5):389-93. Disponível em: https://pdfs.semanticscholar. org/0fb2/1f2a33ed2c06dda13979a24b72ee3ea9b9e7.pdf

11. Sutton S, Link T, Makic MBF. A quality improvement project for safe and effective patient positioning during robot-assisted surgery. AORN J. 2013;97(4):448-56. http://dx.doi.org/10.1016/j.aorn.2013.01.014

12. Malagutti W, Bonfim IM, Eds. Enfermagem em centro cirúrgico: atualidades e perspectivas no ambiente cirúrgico. $3^{\mathrm{a}} \mathrm{ed}$. São Paulo: Martinari; 2013.

13. Bennicoff G. Perioperative care of the morbidly obese patient in the lithotomy position. AORN J [Internet]. 2010 [citado em 10 out. 2017];92(3):297-312. Disponível em: https://aornjournal. onlinelibrary.wiley.com/doi/abs/10.1016/j.aorn.2010.04.016 https:// doi.org/10.1016/j.aorn.2010.04.016

14. World Union of Wound Healing Societies. 0 papel das coberturas na prevenção da lesão por pressão [Internet]. Londres: Wounds International; 2016 [citado em 30 set. 2017]. Disponível em: http:// sobende.org.br/pdf/WUHS\%202016/08.pdf

15. Menezes S, Rodrigues R, Tranquada R, Müller S, Gama K, Manso T. Lesões decorrentes do posicionamento para cirurgia: incidência e fatores de risco. Acta Med Port [Internet]. 2013 [citado em 22 out. 2017];26(1):12-6. Disponível em: http://www.actamedicaportuguesa. com/revista/index.php/amp/article/viewFile/4006/3204

16. Ursi ES, Galvão CM. Ocorrência de úlcera por pressão em pacientes submetidos a cirurgias eletivas. Acta Paul Enferm [Internet]. 2012 [citado em 02 jun. 2017];25(5):653-9. Disponível em: http://www2. unifesp.br/acta/pdf/v25/n5/v25n5a2.pdf http://dx.doi.org/10.1590/ S0103-21002012005000023

17. Costa IG. Incidence of pressure ulcer in regional hospitals of Mato Grosso, Brazil. Rev Gaúcha Enferm [Internet]. 2010 dez. [citado em 31 out. 2017];31(4):693-700. Disponível em: http://www.scielo.br/ scielo.php?script=sci_arttext\&pid=S1983-14472010000400012 http://dx.doi.org/10.1590/S1983-14472010000400012

18. Lindgren $M$, Unosson $M$, Krantz A, Ek A. Pressure ulcer risk factors in patients undergoing surgery. J Adv Nurs. 2005;50(6):605-12. https://doi.org/10.1111/j.1365-2648.2005.03441.x

19. Engels D, Austin M, McNichol L, Fencl J, Gupta S, Kazi H. Pressure ulcers: factors contributing to their development in the OR. AORN J [Internet]. 2016 mar. [citado em 27 out. 2017];103(3):271-81. Disponível em: https://aornjournal. onlinelibrary.wiley.com/doi/abs/10.1016/j.aorn.2016.01.008 https://doi.org/10.1016/j.aorn.2016.01.008 